\title{
Immune checkpoint inhibitor combined with anti-angiogenesis agent inhibits metastasis of advanced adenoid cystic carcinoma of the tongue base to the lung: a case report
}

\author{
Ke Zhang ${ }^{\# \wedge}$, Hui Wang", Juemin Fang, Qing Xu \\ Department of Oncology, Shanghai Tenth People's Hospital, TongJi Cancer Center, School of Medicine, Tongji University, Shanghai, China \\ \#These authors contributed equally to this work. \\ Correspondence to: Juemin Fang; Qing Xu. Department of Oncology, Shanghai Tenth People's Hospital, TongJi Cancer Center, School of Medicine, \\ Tongji University, 301 Middle Yanchang Road, Jing'an, Shanghai 200072, China. Email: fjmholmes@163.com; xuqingmd@aliyun.com.
}

\begin{abstract}
Patients diagnosed with advanced adenoid cystic carcinoma (ACC) with metastasis to the lung generally have poor prognosis when they exhibit resistance to conventional therapies. Programmed celldeath protein 1 (PD-1) inhibitors, a type of Immune checkpoint inhibitors (ICI), have shown good response in the treatment of various types of malignant tumors; however, objective response rates of monotherapy for advanced ACC are low. Anlotinib, a novel, orally managed tyrosine kinase inhibitor, that targets vascular endothelial growth factor receptor (VEGFR), fibroblast growth factor receptor (FGFR), platelet-derived growth factor receptor (PDGFR), and c-kit, has appeared great adequacy in treating numerous sorts of malignant tumors, particularly tumors with lung metastases. Here, we have presented a case of refractory ACC with lung metastases that was reduced after combinatorial treatment using the immune checkpoint inhibitor (ICI) toripalimab and anti-angiogenesis agent anlotinib. The patient achieved a reduction in lung metastases by chest computed tomography (CT) examination, with an outcome of stable disease (SD) of 5 months, a significant decrease in the levels of peripheral blood cytokines interleukin 6 (IL-6) and tumor necrosis factor- $\alpha(\mathrm{TNF}-\alpha)$, as well as good tolerance without noteworthy unfavorable reactions, indicating that the combined therapy of toripalimab and anlotinib may be utilized in the management of advanced ACC.
\end{abstract}

Keywords: Advanced adenoid cystic carcinoma (advanced ACC); toripalimab; anlotinib; case report

Submitted Jun 21, 2021. Accepted for publication Aug 11, 2021.

doi: $10.21037 /$ atm-21-3426

View this article at: https://dx.doi.org/10.21037/atm-21-3426

\section{Introduction}

Adenoid cystic carcinoma (ACC) is an extremely rare and threatening epithelial tumor emerging in salivary glands and other sites. It makes up around $1-2 \%$ of all malignant head and neck tumors (1). Hard plate is the most common intraoral site for minor salivary gland tumors, the following spot is the base of the tongue (2), which comprises $30 \%$ of such cases (3). The occurrence of ACC of the base of the tongue is extremely uncommon, making up as much as $0.1 \%$ of all malignant tumors of the head and neck (4). Regardless of the site, ACC is characterized by $\mathrm{t}(6 ; 9)$ translocation which commonly results in a fusion of the transcription factor genes MYB and NFIB (5-7). In addition, ACC is also associated with the mutations in common pathways, including those involved in chromatin remodeling, DNAdamage/checkpoint signaling, FGF-IGF-PI3K signaling, and NOTCH signaling $(8,9)$. The term "wolf in a sheep's skin" characterizes ACC because of its indolence for progressing submucosally mainly without symptoms,

\footnotetext{
$\wedge$ ORCID: 0000-0002-4037-1952.
} 


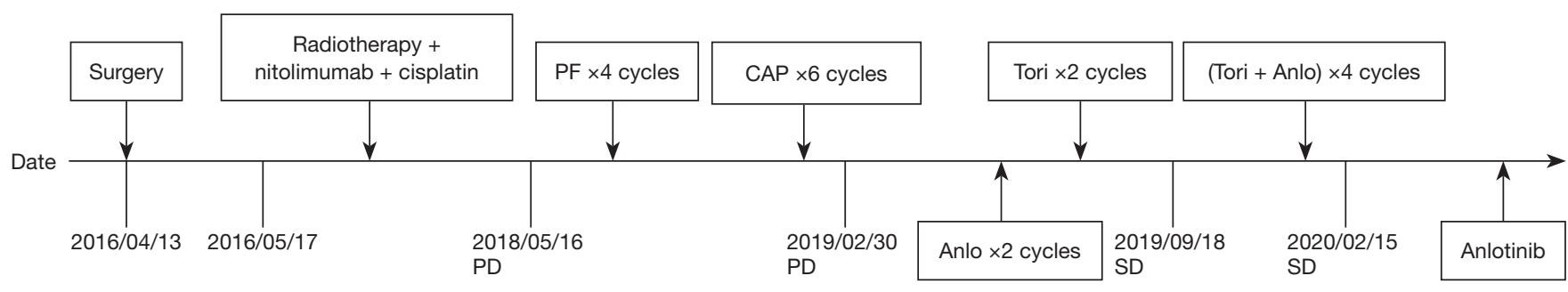

Figure 1 Time line of interventions and patient outcomes.

leading to a high incidence of local recurrence and distant metastasis (1). Thus, around $75 \%$ of ACCs of the tongue base have developed beyond stage T3 when they got diagnosed (10).

Numerous researches have recommended various therapies for ACC, such as surgical resection, radiotherapy, chemotherapy, and combined therapy. Unfortunately, an ideal treatment strategy for dealing with progressed cases has not yet been established. Therapy for ACC most often includes combined wide resection with postoperative X-ray therapy (11). Complete surgical resection is the standard treatment for advanced ACC. Nevertheless, surgical approaches require wide margins and may bring about the debilitation of both swallowing and speech functions. Furthermore, ACC has been recognized as a radioresistant tumor $(12,13)$. Some researchers have proposed that the resistance to radiotherapy for progressed and inoperable ACC tumors predicts the worst prognosis $(4,14,15)$. There is also a lack of evidence on the efficacy of chemotherapy for ACC. In spite of the fact that specific chemoradiotherapy is frequently performed, its therapeutic improvement $\mathrm{t}$ is not ideal (4). Recently, Tchekmedyian et al. demonstrated a $15.6 \%$ response rate in patients with recurrent or metastatic ACC treated with the multitargeted tyrosine kinase inhibitor Lenvatinib. Unfortunately, Lenvatinib was very difficult to tolerate (16). Hence, few other choices can be selected by most advanced ACC patients when they show resistance to customary therapies. Therefore, it is high time to search for ideal medications to lengthen survival time and improve the quality of life of advanced ACC patients. Herein, we have reported a case of advanced ACC of the base of the tongue that showed a reduction in lung lesions under the treatment of toripalimab combined with anlotinib, with an outcome of stable disease (SD) for 5 months.

We present the following article in accordance with the CARE reporting checklist (available at https://dx.doi. org/10.21037/atm-21-3426).

\section{Case presentation}

A 53-year-old male diagnosed with tongue cancer underwent surgery in April 2016. His pathology revealed ACC of the tongue with a pathological staging of pT4N1M0. Regular follow-ups were conducted to monitor disease progress. The timeline of interventions and outcomes of the case are shown in Figure 1.

The patient commenced postoperative radiotherapy with a dose of DT: 6,880 cGy/215 cGy/32 Fx on 17 May 2016. On 21 May 2016, he received simultaneous radiotherapy and chemotherapy of $100 \mathrm{mg}$ weekly injection of nimotuzumab combined with $40 \mathrm{mg}$ of cisplatin. Radiotherapy was suspended due to the patient experiencing throat pain and discomfort when the radiotherapy dose reached planning target volume (PTV), DT: 2,580 cGy/12 Fx. Radiotherapy was resumed on 20 June 2016. Bone scanning performed on 7 July 2016 revealed multiple bone metastases. The patient then received the treatment of zoledronic acid for a year. $\mathrm{He}$ refused follow-up courses of radiotherapy on 13 July 2016. Chest computed tomography (CT) scanning on 20 March 2018 showed multiple nodules in the lingular segment of the upper-left lobe as well as multiple enlarged lymph nodes within the mediastinum, both indicating new metastases. A lung biopsy was performed on 2 May 2018. Pathological diagnosis confirmed that the lung metastases were derived from ACC (Figure 2). The patient then received 4 cycles of PF [5-FU $750 \mathrm{mg}\left(500 \mathrm{mg} / \mathrm{m}^{2}\right) \mathrm{d} 1-\mathrm{d} 3$ + cisplatin $60 \mathrm{mg}$ $\left.\left(75 \mathrm{mg} / \mathrm{m}^{2}\right) \mathrm{d} 1-\mathrm{d} 2 \mathrm{q} 3 \mathrm{w}\right]$ regimen starting 16 May 2018. The follow-up results after 2 months of chemotherapy showed disease progression (PD). He then was treated with 6 cycles of cyclophosphamide combined with epirubicin and cisplatin (CAP) regimen starting from 30 August 2018. A subsequent CT scan again indicated PD.

The patient then received an anlotinib (12 mg, d1-d14, $\mathrm{q} 3 \mathrm{w}$ ) regimen starting 16 April 2019. The CT examination after 2 cycles of anlotinib showed lung lesions in the lateral segment of the middle-right lobe and upper-left lobe, 

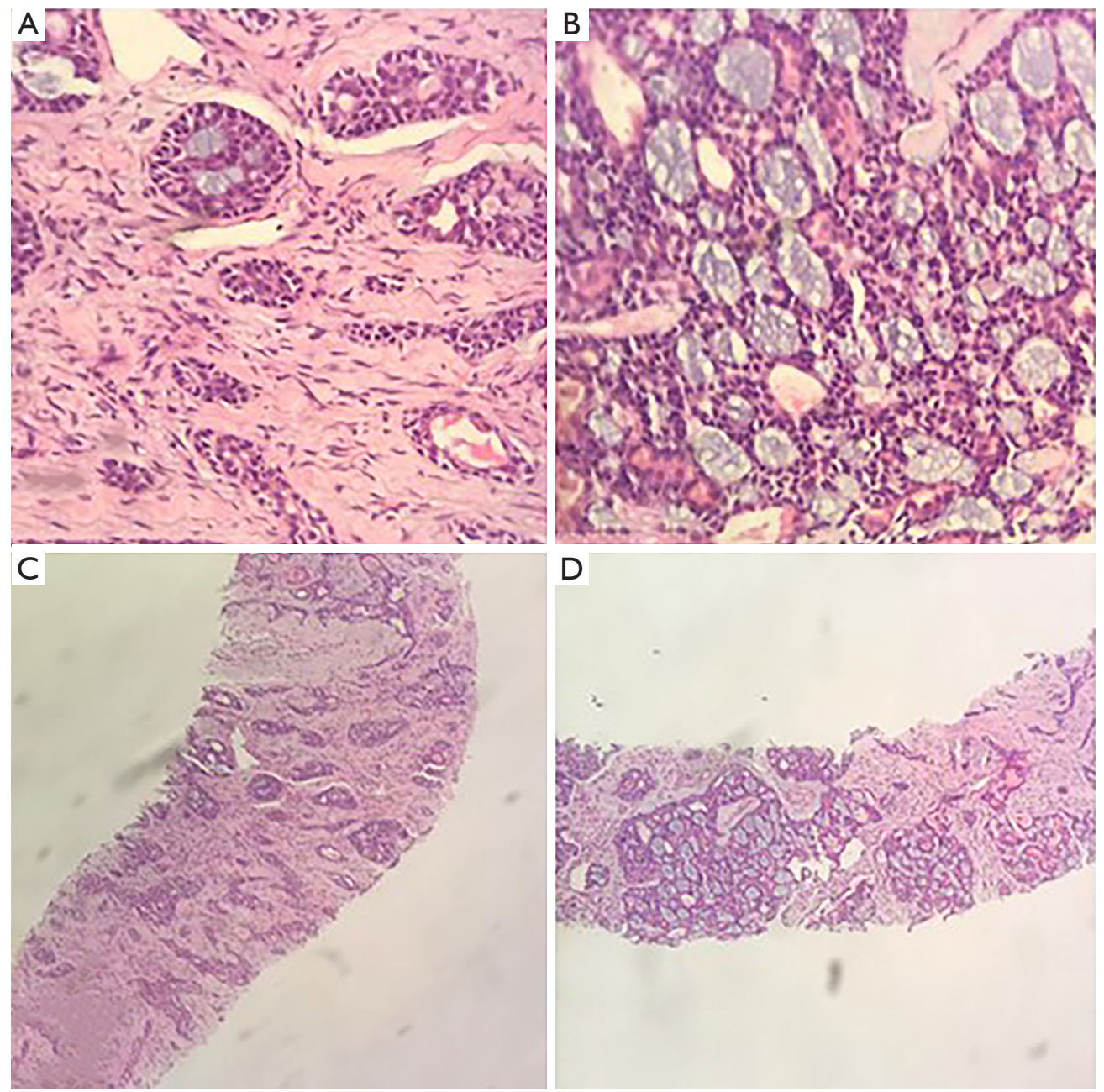

Figure 2 Representative images of $\mathrm{H} \& \mathrm{E}$ staining in adenoid cystic carcinoma-derived lung metastases from a lung biopsy. Images (A) and (B) were obtained at 40× magnification. Images (C) and (D) were obtained at 10× magnification. H\&E, hematoxylin and eosin.

indicating an outcome of PD (Figure $3 A$ ). Treatment was then switched to toripalimab (3 $\mathrm{mg} / \mathrm{kg}, \mathrm{Q} 3 \mathrm{w}$ ) starting from 1 July 2019. After 2 cycles of toripalimab, CT examination showed slight enlargement of the lung tumor, indicating an outcome of PD (Figure 3B). Laboratory examinations showed no specific evaluated tumor markers. Results of genetic testing [next generation sequencing (NGS)] revealed no specific gene mutations for targeted therapy (Table 1). Considering the associated studies with regard to the effectiveness of combination of immune checkpoint inhibitors (ICIs) and anti-angiogenesis, the patient was administered an exploratory treatment regimen consisting of toripalimab $(3 \mathrm{mg} / \mathrm{kg}, \mathrm{q} 3 \mathrm{w})$ and anlotinib $(12 \mathrm{mg}$, d1$\mathrm{d} 14, \mathrm{q} 3 \mathrm{w})$ starting from 18 September 2019. Results of Chest CT examined after 2 cycles and 4 cycles of treatment showed a reduction in lung lesions, with an outcome of SD for 5 months (Figure 3C,3D). The patient suspended toripalimab treatment in February 2020 and continued with anlotinib monotherapy, which has been maintained to date. Chest CT after 6 cycles and 9 cycles of anlotinib monotherapy showed SD (Figure 3E, 3F). Peripheral blood cytokine examinations showed a significant downward trend of interleukin 6 (IL-6) and tumor necrosis factor- $\alpha$ (TNF- $\alpha$ ) (Figure 4). The patient showed good tolerance to this treatment regimen and no severe adverse reactions were observed during treatment.

At the time of writing this report, the patient was still alive and experiencing a good quality of life. All procedures performed in studies involving human participants were in accordance with the ethical standards of the institutional 

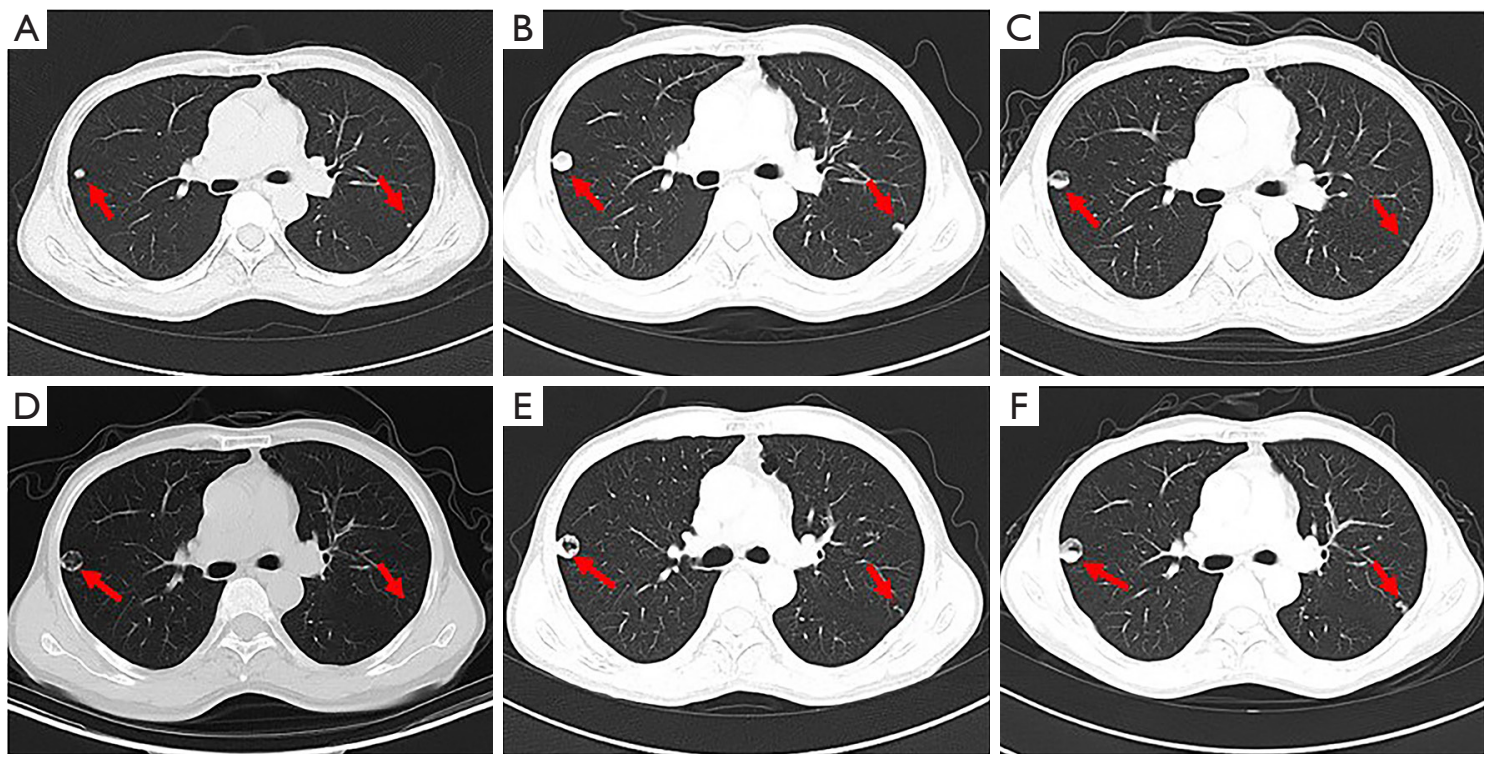

Figure 3 Chest CT (A-F). (A) Lung lesions in the lateral segment of the right middle lobe and the left upper lobe exhibiting malignant metastases. (B) Slight enlargement of lung lesions in the lateral segment of the right middle lobe and the left upper lobe. (C) Chest CT after 2 cycles of combined therapy of toripalimab and anlotinib revealed reduction in lung metastases, with an outcome of SD. (D) Chest CT after 4 cycles of combined therapy of toripalimab and anlotinib indicated a significant reduction in lung metastases, supporting an outcome of SD. (E) Chest CT after 6 cycles of the monotherapy of anlotinib showed slight enlargement of lung lesions. (F) Chest CT after 9 cycles of monotherapy of anlotinib showed significant improvement of lung lesions. The red arrows indicate metastatic lesions in the lungs. CT, computed tomography; SD, stable disease.

Table 1 Mutations detected in the patient

\begin{tabular}{lc}
\hline Genes & Results \\
\hline UGT1A1 & p.G1R \\
& c.211G>A \\
& rs $4148323^{*} 6$ \\
ALK & None \\
$B R A F$ & None \\
$B R C A 1$ & None \\
$B R C A 2$ & None \\
CD274 (PD-L1) & None \\
EGFR & None \\
$K R A S$ & None \\
NRAS & None \\
\hline
\end{tabular}

and/or national research committee(s) and with the Helsinki Declaration (as revised in 2013). Written informed consent was obtained from the patient for publication of this case report and accompanying images. A copy of the written

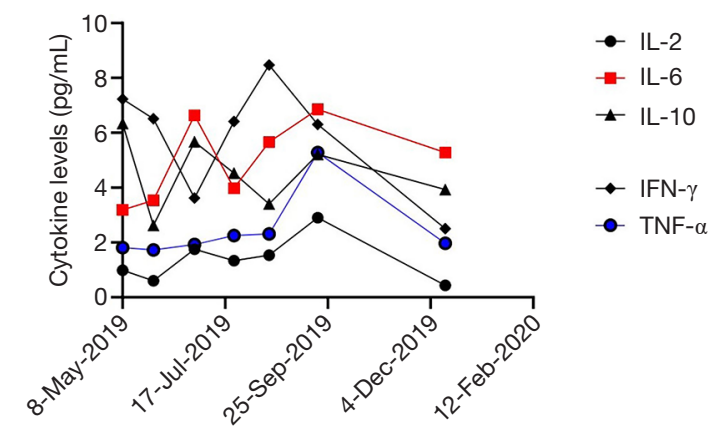

Figure 4 Changes in peripheral blood cytokine levels of the patient. IL-2, interleukin 2 ; IFN- $\gamma$, interferon $\gamma$; TNF- $\alpha$, tumor necrosis factor- $\alpha$.

consent is available for review by the editorial office of this journal.

\section{Discussion}

To date, no researcher has ever studied the clinical use of ICI combined with anti-angiogenesis agents in the treatment of 
advanced ACC of the tongue base. As far as we know, this was the first report of a patient with chemorefractory and radiorefractory advanced ACC of the base of the tongue who showed lung metastasis shrinkage under the treatment of toripalimab combined with anlotinib.

Cancer immunotherapy, aiming to improve antitumour immune responses with immune system has become a powerful clinical strategy for treating cancer. Immunotherapies fall into the following several categories, including checkpoint inhibitors, lymphocyte-activating cytokines, CAR T cells and other cellular therapies, antibodies against co-stimulatory receptors, cancer vaccines, oncolytic viruses and bispecific antibodies (17). Evidence has showed that tumor microenvironment (TME) is a predictive factor of response to immunotherapy, along with imaging results (18). Compared with conventional therapies, such as radiotherapy, chemotherapy and targeted therapy, immunotherapy can induce immune system to eliminate abnormal cells by checking cell surface for specific markers or antigens in spite of what status they are in (active or dormant) (18). In addition, circulating tumor cells (CTCs) and disseminated tumor cells (DTCs) in immunosuppressive tumor microenvironment (TME) are more likely to be monitored and captured by cells such as macrophages, natural killer (NK) cells and cytotoxic T lymphocytes (CTLs), suggesting that immunotherapy is promising in controlling metastasis (19). Among those immunotherapies, therapy targeting programmed death-1 (PD-1) receptor has shown effective and durable clinical responses in patients with various cancer types, such as malignant melanoma, non-small cell lung cancer, renal cell carcinoma, classical Hodgkin lymphoma, and recurrent or metastatic head and neck squamous cell carcinoma $(17,18)$.

Toripalimab, a programmed cell-death protein 1 (PD-1) blockade, is one type of various ICIs. Tumor cells can evade the immune surveillance mediated with $T$ cell by utilizing the inhibitory $\mathrm{PD}-1 /$ programmed cell death 1 ligand 1 (PD-L1) immune checkpoint. Thus, antibodies blocking $\mathrm{PD}-1 / \mathrm{PD}-\mathrm{L} 1$ can induce durable clinical efficacy on cancer cells (20). Toripalimab can restore the $\mathrm{T}$ cells with cytotoxicity effect against cancer cells by blocking the PD-1 immune checkpoint. Studies have revealed that ICI show good efficacy on the treatment for a variety of malignant tumors, including solid tumors (21-25). As a monotherapy for advanced ACC, however, the objective response rate is low (26). Researchers have found that the proportions of CD8+, GrB+, TIL, CD1a, and CD83 of ACC patients are low and the positivity for CTLA-4 and PD-1 in its immune microenvironment is deficient (27). This immune scenario causes tumor cells to escape the immune system and may partially bring the poor efficacy of ICI on ACC patients to light. In spite of its anti-tumor effectiveness, ICI also has a variety of long-term toxic reactions, such as allergic reactions, a spectrum of inflammatory side effects, or immune-related adverse events (irAEs) targeting not only the tumor lesions, but also the normal tissues (28).

Anlotinib is a small-molecule multi-target tyrosine kinase inhibitor (TKI) that inhibits tumor development and angiogenesis by strongly inhibiting numerous targets including vascular endothelial growth factor receptor (VEGFR), fibroblast growth factor receptor (FGFR), plateletderived growth factor receptor (PDGFR), and c-Kit (29). Furthermore, studies have revealed that anlotinib can improve the efficacy of PD-1 blockade synergistically (30). Angiogenesis is the process of forming new blood vessels from pre-existing ones and it is thought to be very important for tumor growth, invasion, and metastasis. VEGF and other angiogenic factors are critical in tumor angiogenesis (31). The overexpression of angiogenic factors has an negative impact on the survival of oral cancer patients $(26,32)$. Anlotinib inhibits tumor angiogenesis and cause tumor cells to lose some of their activities (33-35). Anlotinib has been shown in clinical trials to significantly extend the survival duration of advanced cancer patients, including those with squamous cell lung cancer (SCLC), metastatic renal cell carcinoma (mRCC), and soft tissue sarcoma (STS) (36-39). Nevertheless, in this case, we did not observe an ideal response of the ACC patient to anlotinib monotherapy.

Relationships between angiogenesis and immunity in tumors are intricate. A growing body of evidence have showed that tumor microenvironment (TME) plays an essential role in the growth and invasion of tumor and its associated anti-tumor therapies targeted primarily on T cells (40). In our case, Toripalimab can renew the cytotoxicity effect of the $T$ cells against cancer cells in the TME. With a reasonable dose, Anlotinib, the Anti-angiogenesis targeted drugs can also normalize the tumor vasculature and this change can also reduce immunosuppression induced by Tregs and regulatory B cells and promote antitumor immunity by enhancing the uptake of antigen presentation in dendritic cells, M1-associated macrophages and activation of cytotoxic $\mathrm{CD} 8^{+} \mathrm{T}$ cells, thus synergizing with ICI (41). A recent study revealed that anlotinib could increase the infiltration of innate immune cells, which included natural killer (NK) cells and antigen-presenting cells (APC) (30). Furthermore, researchers found that 
anlotinib plus PD-1 therapy dramatically attenuated tumor growth compared with the PD-1 or anlotinib monotherapy groups and control group, indicating that anlotinib enhances the therapeutic efficacy of PD-1 blockade (30). As reported by IMpower150, as first-line treatment for metastatic NSCLC, atezolizumab plus bevacizumab plus chemotherapy significantly increases patient progressionfree survival (PFS) and overall survival (OS), regardless of PD-L1 expression or EGFR or ALK mutation status (42). A previous study on advanced hepatocellular carcinoma (HCC) found that the combination of atezolizumab and bevacizumab results in a $32 \%$ overall response rate (ORR) and a median PFS of 14.9 months, both of which are huge improvements (43). SHR-1210, a PD-1 inhibitor, was also discovered to obtain a $50 \%$ ORR in patients with advanced HCC when combined with the small molecule anti-angiogenesis inhibitor apatinib (44). These data imply that anti-angiogenic medicines have an immunomodulatory effect, which could help ICI work more effectively.

Pharmaceutical care, defined as the responsible provision of drug therapy aiming at improving a patient's quality of life, is also an essential part of the combination therapy of immune checkpoint inhibitors and anti-angiogenesis drugs (45). For now, there are no definite pharmaceutical care standards for this combination therapy. However, according to the toxicity of the two types of drugs, patients need to use anti-allergy drugs preventively during administration, such as promethazine hydrochlorine. In addition, routine monitoring of thyroid function, liver and kidney function, blood pressure, hepatitis B indicators after administration is also indispensable (46). In this case, the patient routinely used promethazine hydrochlorine during the administration and no serious allergic reactions occurred. There was also no significant anomaly in the monitoring results of the above indicators after each course of the combination treatment, suggesting that the patient had good tolerance to this combined therapy.

Previous studies have demonstrated that anti-angiogenesis and ICI therapy has a synergetic enhanced inhibitory effect on various cancers. One of the main issues that needs to be addressed is how to optimize the doses and regimens of ICI and anti-angiogenesis in the combination therapy. In this case, the ICI toripalimab in combination with anti-angiogenic agent anlotinib was administered for the treatment of the patient with refractory advanced ACC of the base of the tongue. The patient showed a reduction in lung metastases by chest CT examinations with an outcome of SD for 5 months, a significant decrease in the levels of peripheral blood cytokines IL- 6 and TNF- $\alpha$, as well as good tolerance without significant adverse reactions, suggesting that ICI plus anti-angiogenesis would be a promising strategy to improve ACC patients' prognosis.

\section{Patient perspective}

When I was diagnosed with advanced ACC at my tongue base in 2016, I didn't immediately feel frustrated. I thought modern medicine is so developed that there will always be a way to cure my disease. Shortly after the diagnosis, I underwent the resection of the tumor mass. As soon as I recovered from the operation, the doctor gave me the treatment of radiotherapy combined with chemotherapy. With the increase of radiation dose, I developed symptoms of sore throat, which was a very difficult time. Unfortunately, the tumor cells metastasized to my bones and lungs. With the comfort of my family and the advice of my doctor, I tried a variety of chemotherapy options and even tried monotherapy of anlotinib and toripalimab. However, every time I did the treatment assessment, the result was always PD, which made me suffer both psychological and physical pain.

When my doctor advised me to try a combination of anlotinib and toripalimab, I hesitated, because both drugs were expensive and my illness had not improved a lot, but I was ultimately persuaded. After two courses of the treatment, I developed symptoms of fatigue, but this is still within my tolerance range, and my indicators were normal. Finally, in an unknown number of assessments, my condition improved, the lesions in my lung shrunk, and this stable state lasted for five months. I don't know how to describe the excitement at that time. Although my later treatment was affected by the epidemic, I believe this combination treatment is effective for me. I will continue to listen to the doctor's advice and actively cooperate with the treatment.

\section{Acknowledgments}

Funding: This work was supported by the National Natural Science Foundation of China (81702311, 81772905, 81803090, and 81902896) and the Shanghai Science and Technology Committee (18DZ1910102).

\section{Footnote}

Reporting Checklist: The authors have completed the 
CARE reporting checklist. Available at https://dx.doi. org/10.21037/atm-21-3426

Conflicts of Interest: All authors have completed the ICMJE uniform disclosure form (available at https://dx.doi. org/10.21037/atm-21-3426). The authors have no conflicts of interest to declare.

Ethical Statement: The authors are accountable for all aspects of the work in ensuring that questions related to the accuracy or integrity of any part of the work are appropriately investigated and resolved. All procedures performed in studies involving human participants were in accordance with the ethical standards of the institutional and/or national research committee(s) and with the Helsinki Declaration (as revised in 2013). Written informed consent was obtained from the patient for publication of this case report and accompanying images. A copy of the written consent is available for review by the editorial office of this journal.

Open Access Statement: This is an Open Access article distributed in accordance with the Creative Commons Attribution-NonCommercial-NoDerivs 4.0 International License (CC BY-NC-ND 4.0), which permits the noncommercial replication and distribution of the article with the strict proviso that no changes or edits are made and the original work is properly cited (including links to both the formal publication through the relevant DOI and the license). See: https://creativecommons.org/ licenses/by-nc-nd/4.0/.

\section{References}

1. Kim KH, Sung MW, Chung PS, et al. Adenoid cystic carcinoma of the head and neck. Arch Otolaryngol Head Neck Surg 1994;120:721-6.

2. Khafif A, Anavi Y, Haviv J, et al. Adenoid cystic carcinoma of the salivary glands: a 20-year review with long-term follow-up. Ear Nose Throat J 2005;84:662, 664-7.

3. Spiro RH, Huvos AG, Strong EW. Adenoid cystic carcinoma of salivary origin. A clinicopathologic study of 242 cases. Am J Surg 1974;128:512-20.

4. Goepfert H, Giraldo AA, Byers RM, et al. Salivary gland tumors of the base of the tongue. Arch Otolaryngol 1976;102:391-5.

5. Stenman G, Sandros J, Dahlenfors R, et al. 6q- and loss of the $\mathrm{Y}$ chromosome--two common deviations in malignant human salivary gland tumors. Cancer Genet Cytogenet 1986;22:283-93.

6. Mertens F, Johansson B, Fioretos T, et al. The emerging complexity of gene fusions in cancer. Nat Rev Cancer 2015;15:371-81.

7. Persson M, Andrén Y, Mark J, et al. Recurrent fusion of MYB and NFIB transcription factor genes in carcinomas of the breast and head and neck. Proc Natl Acad Sci U S A 2009;106:18740-4.

8. Ho AS, Kannan K, Roy DM, et al. The mutational landscape of adenoid cystic carcinoma. Nat Genet 2013;45:791-8.

9. Stephens PJ, Davies HR, Mitani Y, et al. Whole exome sequencing of adenoid cystic carcinoma. J Clin Invest 2013;123:2965-8.

10. Takayama K, Kato T, Nakamura T, et al. Proton Beam Therapy Combined with Intra-Arterial Infusion Chemotherapy for Stage IV Adenoid Cystic Carcinoma of the Base of the Tongue. Cancers (Basel) 2019;11:1413.

11. Silverman DA, Carlson TP, Khuntia D, et al. Role for postoperative radiation therapy in adenoid cystic carcinoma of the head and neck. Laryngoscope 2004;114:1194-9.

12. Chen AM, Bucci MK, Weinberg V, et al. Adenoid cystic carcinoma of the head and neck treated by surgery with or without postoperative radiation therapy: prognostic features of recurrence. Int J Radiat Oncol Biol Phys 2006;66:152-9.

13. Mendenhall WM, Morris CG, Amdur RJ, et al. Radiotherapy alone or combined with surgery for adenoid cystic carcinoma of the head and neck. Head Neck 2004;26:154-62.

14. Kokemueller H, Eckardt A, Brachvogel P, et al. Adenoid cystic carcinoma of the head and neck--a 20 years experience. Int J Oral Maxillofac Surg 2004;33:25-31.

15. Sur RK, Donde B, Levin V, et al. Adenoid cystic carcinoma of the salivary glands: a review of 10 years. Laryngoscope 1997;107:1276-80.

16. Tchekmedyian V, Sherman EJ, Dunn L, et al. Phase II Study of Lenvatinib in Patients With Progressive, Recurrent or Metastatic Adenoid Cystic Carcinoma. J Clin Oncol 2019;37:1529-37.

17. Riley RS, June CH, Langer R, et al. Delivery technologies for cancer immunotherapy. Nat Rev Drug Discov 2019;18:175-96.

18. Maleki Vareki S, Garrigós C, Duran I. Biomarkers of response to PD-1/PD-L1 inhibition. Crit Rev Oncol Hematol 2017;116:116-24.

19. Gao S, Yang D, Fang Y, et al. Engineering Nanoparticles 
for Targeted Remodeling of the Tumor Microenvironment to Improve Cancer Immunotherapy. Theranostics 2019;9:126-51.

20. Goodman A, Patel SP, Kurzrock R. PD-1-PD-L1 immune-checkpoint blockade in B-cell lymphomas. Nat Rev Clin Oncol 2017;14:203-20.

21. Kudo T, Hamamoto Y, Kato K, et al. Nivolumab treatment for oesophageal squamous-cell carcinoma: an open-label, multicentre, phase 2 trial. Lancet Oncol 2017;18:631-9.

22. Doi T, Piha-Paul SA, Jalal SI, et al. Safety and Antitumor Activity of the Anti-Programmed Death-1 Antibody Pembrolizumab in Patients With Advanced Esophageal Carcinoma. J Clin Oncol 2018;36:61-7.

23. Shah MA, Kojima T, Hochhauser D, et al. Efficacy and Safety of Pembrolizumab for Heavily Pretreated Patients With Advanced, Metastatic Adenocarcinoma or Squamous Cell Carcinoma of the Esophagus: The Phase 2 KEYNOTE-180 Study. JAMA Oncol 2019;5:546-50.

24. Huang J, Xu B, Mo H, et al. Safety, Activity, and Biomarkers of SHR-1210, an Anti-PD-1 Antibody, for Patients with Advanced Esophageal Carcinoma. Clin Cancer Res 2018;24:1296-304.

25. Tang Y, Ou Z, Yao Z, et al. A case report of immune checkpoint inhibitor nivolumab combined with antiangiogenesis agent anlotinib for advanced esophageal squamous cell carcinoma. Medicine (Baltimore) 2019;98:e17164.

26. Lin B, Song X, Yang D, et al. Anlotinib inhibits angiogenesis via suppressing the activation of VEGFR2, PDGFR $\beta$ and FGFR1. Gene 2018;654:77-86.

27. Ferrara N, Hillan KJ, Gerber HP, et al. Discovery and development of bevacizumab, an anti-VEGF antibody for treating cancer. Nat Rev Drug Discov 2004;3:391-400.

28. Kennedy LB, Salama AKS. A review of cancer immunotherapy toxicity. CA Cancer J Clin 2020;70:86-104.

29. Han B, Li K, Wang Q, et al. Effect of Anlotinib as a Third-Line or Further Treatment on Overall Survival of Patients With Advanced Non-Small Cell Lung Cancer: The ALTER 0303 Phase 3 Randomized Clinical Trial. JAMA Oncol 2018;4:1569-75.

30. Yang Y, Li L, Jiang Z, et al. Anlotinib optimizes antitumor innate immunity to potentiate the therapeutic effect of PD-1 blockade in lung cancer. Cancer Immunol Immunother 2020;69:2523-32.

31. Zhao SF, Yang XD, Lu MX, et al. Prognostic significance of VEGF immunohistochemical expression in oral cancer: a meta-analysis of the literature. Tumour Biol
2013;34:3165-71.

32. Lin YW, Huang ST, Wu JC, et al. Novel HDGF/HIF-1 $\alpha$ / $\mathrm{VEGF}$ axis in oral cancer impacts disease prognosis. BMC Cancer 2019;19:1083.

33. He C, Wu T, Hao Y. Anlotinib induces hepatocellular carcinoma apoptosis and inhibits proliferation via Erk and Akt pathway. Biochem Biophys Res Commun 2018;503:3093-9.

34. Liang L, Hui K, Hu C, et al. Autophagy inhibition potentiates the anti-angiogenic property of multikinase inhibitor anlotinib through JAK2/STAT3/VEGFA signaling in non-small cell lung cancer cells. J Exp Clin Cancer Res 2019;38:71.

35. Han B, Li K, Zhao Y, et al. Anlotinib as a third-line therapy in patients with refractory advanced non-smallcell lung cancer: a multicentre, randomised phase II trial (ALTER0302). Br J Cancer 2018;118:654-61.

36. Sun Y, Du F, Gao M, et al. Anlotinib for the Treatment of Patients with Locally Advanced or Metastatic Medullary Thyroid Cancer. Thyroid 2018;28:1455-61.

37. Zhou AP, Bai YX, Song Y, et al. Anlotinib versus sunitinib as first line treatment for metastatic renal cell carcinoma (mRCC): preliminary results from a randomized phase II clinical trial. J Clin Oncol 2016;34:abstr 4565.

38. Chi Y, Fang Z, Hong X, et al. Safety and Efficacy of Anlotinib, a Multikinase Angiogenesis Inhibitor, in Patients with Refractory Metastatic Soft-Tissue Sarcoma. Clin Cancer Res 2018;24:5233-8.

39. Mosconi C, de Arruda JAA, de Farias ACR, et al. Immune microenvironment and evasion mechanisms in adenoid cystic carcinomas of salivary glands. Oral Oncol 2019;88:95-101.

40. Hinshaw DC, Shevde LA. The Tumor Microenvironment Innately Modulates Cancer Progression. Cancer Res 2019;79:4557-66.

41. Huang Y, Goel S, Duda DG, et al. Vascular normalization as an emerging strategy to enhance cancer immunotherapy. Cancer Res 2013;73:2943-8.

42. Socinski MA, Jotte RM, Cappuzzo F, et al. Atezolizumab for First-Line Treatment of Metastatic Nonsquamous NSCLC. N Engl J Med 2018;378:2288-301.

43. Pishvaian MJ, Lee MS, Ryoo B, et al. Updated safety and clinical activity results from a Phase Ib study of atezolizumab + bevacizumab in hepatocellular carcinoma. Ann Oncol 2018;29:mdy424.028.

44. Xu J, Zhang Y, Jia R, et al. Anti-PD-1 Antibody SHR-1210 Combined with Apatinib for Advanced Hepatocellular Carcinoma, Gastric, or Esophagogastric Junction Cancer: 
An Open-label, Dose Escalation and Expansion Study. Clin Cancer Res 2019;25:515-23.

45. Hepler CD, Strand LM. Opportunities and responsibilities in pharmaceutical care. Am J Hosp Pharm 1990;47:533-43.

Cite this article as: Zhang K, Wang H, Fang J, Xu Q. Immune checkpoint inhibitor combined with anti-angiogenesis agent inhibits metastasis of advanced adenoid cystic carcinoma of the tongue base to the lung: a case report. Ann Transl Med 2021;9(16):1353. doi: 10.21037/atm-21-3426
46. Moltó-Puigmartí C, Vonk R, van Ommeren G, et al. A logic model for pharmaceutical care. J Health Serv Res Policy 2018;23:148-57.

(English Language Editor: J. Jones) 